\title{
Dislocation core reconstruction induced by carbon segregation in bcc iron
}

\author{
Lisa Ventelon, ${ }^{1, *}$ B. Lüthi, ${ }^{1}$ E. Clouet, ${ }^{1}$ L. Proville,${ }^{1}$ B. Legrand,${ }^{1}$ D. Rodney, ${ }^{2}$ and F. Willaime ${ }^{1}$ \\ ${ }^{1}$ CEA, DEN, Service de Recherches de Métallurgie Physique, F-91191 Gif-sur-Yvette, France \\ ${ }^{2}$ Institut Lumière Matière, Université Lyon 1, CNRS, UMR 5306, F-69622 Villeurbanne, France \\ (Received 24 October 2014; revised manuscript received 8 June 2015; published 29 June 2015)
}

\begin{abstract}
The relative stability of dislocation core configurations in body-centered-cubic metals is profoundly modified by the presence of solutes. Considering the $\mathrm{Fe}(\mathrm{C})$ system, we demonstrate by using density functional theory that carbon atoms destabilize the usual easy core to the benefit of the hard core configuration of the screw dislocation, which is unstable in pure metals. The carbon atom is at the center of a regular prism in a cementitelike local environment. The same dislocation core reconstruction is also found with other solutes $(B, N, O)$ and in $W(C)$. This unexpected low-energy configuration induces a strong solute-dislocation attraction, leading to dislocation core saturation by solute atoms, even for very low bulk solute concentrations. This core reconstruction will constitute an essential factor to account for in solute-segregation related phenomena, such as strain aging.
\end{abstract}

DOI: 10.1103/PhysRevB.91.220102

PACS number(s): 61.72.Lk, 71.15.Mb

One of the key objectives to improve our fundamental comprehension of plasticity is to understand how the dislocations responsible for the plastic deformation interact with solute atoms, whose pinning strength produces the hardness required for industrial applications [1,2]. This has led to an active area of research focused on the microscopic mechanisms of solute segregation near dislocations, starting with the seminal work of Cottrell and Bilby [3]. On the modeling side, so far most effort has been devoted to the interaction between solute atoms and dislocations in regions away from the dislocation core where the elastic theory is valid [4-7]. This also justifies representing atomic bonding with semiempirical potentials that are known to reproduce elastic properties with fidelity. The case where a solute atom enters the dislocation core, which can potentially lead to the strongest pinning effect, is much more difficult to study since strong perturbations of the atomic interactions beyond the reach of elasticity are expected. Such studies require an accurate description of atomic bonding, down to the electronic structure level.

Dislocation core effects are prominent in body-centeredcubic (bcc) metals. Most first-principles works to date have considered the energetics of $1 / 2\langle 111\rangle$ screw dislocations in pure metals [8-15] where, after much debate, it is now accepted that the dislocation adopts a symmetrical (or nondegenerate) configuration called the easy core, centered on a triangle of first neighbor $\langle 111\rangle$ atomic columns where helicity is reversed compared to the bulk. Other cores, all unstable in pure bcc metals, are also known: an asymmetrical easy core, a hard core where the three atoms around the core belong to the same (111) plane, and a split core centered in the immediate vicinity of an atomic column [12-14]. Only few first-principles studies have considered substitutional [16-18] and interstitial solutes $[19,20]$. They have shown, for instance, that substitutional solute alloying can stabilize the asymmetrical easy core.

In this Rapid Communication, we investigate the effect of interstitial solutes on $1 / 2\langle 111\rangle$ screw dislocations in bcc metals using density functional theory (DFT) calculations based on the VASP code [21]. We show that, unexpectedly, solute atoms can stabilize the hard core configuration with a strong binding

*lisa.ventelon@cea.fr energy, profoundly altering our traditional view of plasticity in bcc metals. We focus here on the $\mathrm{Fe}(\mathrm{C})$ system because of its practical interest as the basis of ferritic steels, where interstitial carbon atoms are known experimentally to strongly interact with the dislocations [22-24], but we have observed the same effects with other solutes $(\mathrm{B}, \mathrm{N}, \mathrm{O})$, as well as in other metals, in particular, W(C). We have also obtained the same phenomenology with two other independent DFT codes, SIESTA [25] and QUANTUM ESPRESSO [26].

We performed spin-polarized calculations using the projector augmented wave pseudopotential scheme [27,28] within the Perdew-Burke-Ernzerhof generalized gradient approximation and a $400 \mathrm{eV}$ kinetic-energy cutoff. We considered a quadrupolar periodic array of dislocation dipoles with a distance between dislocations of $7.5 \sqrt{2 / 3} a_{0}$ in the $\langle 112\rangle$ direction (for a detailed description of the cell geometry, see Ref. [12]), with carbon atoms separated from $1 b$ to $6 b$ along the dislocation line ( $b$ is the dislocation Burgers vector), resulting from $135(+2)$ to $810(+2)$-atom simulation cells. We used a $0.3 \mathrm{eV}$ Hermite Gaussian broadening, and $1 \times 2 \times 16$, $1 \times 2 \times 8,1 \times 2 \times 6,1 \times 2 \times 4,1 \times 2 \times 3$, and $1 \times 2 \times 3$ shifted $k$-point grids for the cell sizes along the dislocation line of $1 b$ to $6 b$. Both dislocations of the dipole are initially relaxed with an easy core, with the cell shape adapted to this configuration [12]. Carbon atoms are then introduced near both dislocations on octahedral interstitial sites, which are the stable interstitial positions in the perfect lattice [29,30], and the atomic positions are relaxed again. Several sites in the vicinity of the dislocation core were investigated but only the two most important ones are presented here: the octahedral positions of the first and second nearest neighbors to the dislocation core, denoted $\mathrm{O}^{(1)}$ and $\mathrm{O}^{(2)}$, respectively [Fig. 1(a)].

Whether the carbon atoms are introduced in an $\mathrm{O}^{(1)}$ or an $\mathrm{O}^{(2)}$ site [see Fig. 1(b)], we find upon energy minimization that the system spontaneously reorganizes and relaxes towards a configuration where the dislocation and the carbon atoms are centered on a hard core position, as illustrated in Fig. 1(c). This unexpected core reconstruction, observed whether the carbon atoms are separated from $1 b$ to $6 b$, although at $4 b$ and beyond the prism is no longer regular but instead distorted, means that the presence of the carbon atoms in the vicinity of the core is sufficient to invert the relative stability between the easy and 


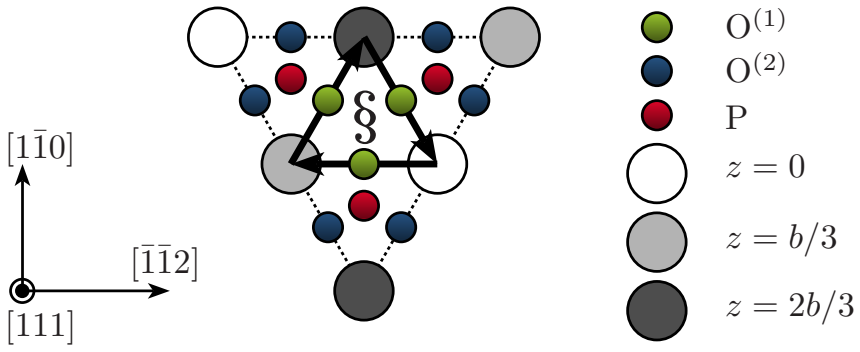

(a)
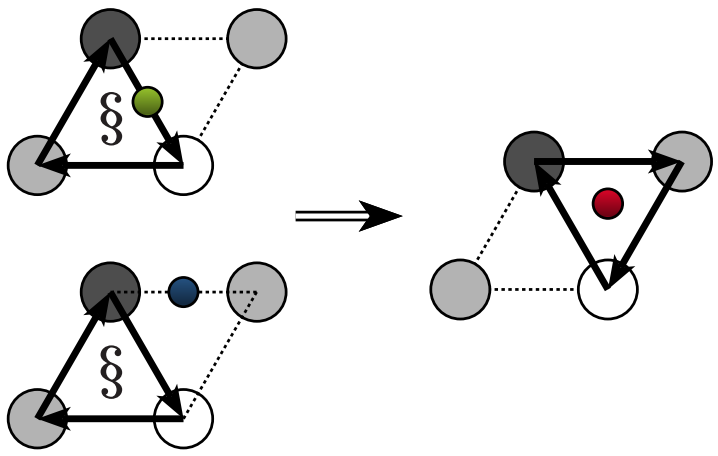

(b)

(c)

FIG. 1. (Color online) (a) Projection on the (111) plane of the iron (in white and gray) and carbon (in color) atomic positions around the dislocation core (represented by the $\S$ symbol): $\mathrm{O}^{(1)}$ (respectively $\mathrm{O}^{(2)}$ ) denotes the octahedral sites, first (respectively second) nearest neighbors to the dislocation core; $\mathrm{P}$ denotes the prismatic sites inside the hard core. (b) Before relaxation of the $\mathrm{O}^{(1)}$ and $\mathrm{O}^{(2)}$ configurations, the dislocation has an easy core configuration. (c) After relaxation, the dislocation core is positioned on a neighboring hard core configuration and the carbon atoms are situated on a $\mathrm{P}$ site. The dislocation core is visualized by its differential displacement map [31] and the iron atomic positions are colored in gray scale according to their (111) plane in the perfect bcc lattice.

hard core structures. Similar calculations with other solute atoms $(\mathrm{B}, \mathrm{N}, \mathrm{O})$ in $\mathrm{Fe}$ have lead to the same reconstruction. In $\mathrm{Fe}$, this inversion of stability is helped by the fact that the hard core has a remarkably low relative energy [12-14], but we also found the same reconstruction in $\mathrm{W}(\mathrm{C})$, where the hard core has a higher relative energy. The solute environment in the hard core therefore controls the inversion of stability.

In the hard core configuration, the three central $\langle 111\rangle$ atomic columns form an array of regular trigonal prisms with a height of $1 b$ (see Fig. 2). The interstitial carbon atom then occupies the center of a prism [P position in Fig. 1(a)] and induces an expansion of the prism in the (111) plane and, if spaced by more than $1 b$, also in the direction of the Burgers vector (see Table I). In fact, the stability of this prism structure may have been anticipated for several reasons. First, the local environment around the carbon atom within the prism, namely, the number of neighbors, atomic distances, and local magnetic moments, are comparable to the octahedral site in the perfect bcc structure. Second, $\mathrm{Fe}_{3} \mathrm{C}$ cementite, the iron carbide that precipitates in slowly cooled supersaturated ferritic alloys, is composed of corner- and edge-sharing trigonal iron prisms centered on carbon atoms [32], and Table I shows that the $\mathrm{Fe}-\mathrm{Fe}$ and $\mathrm{Fe}-\mathrm{C}$ distances within the trigonal prisms are

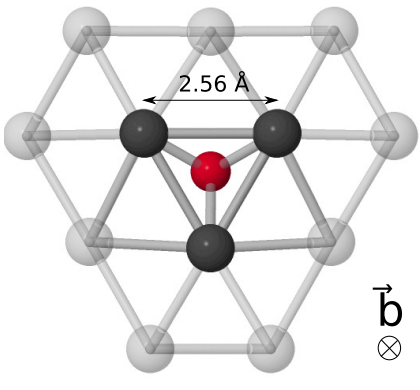

(a)

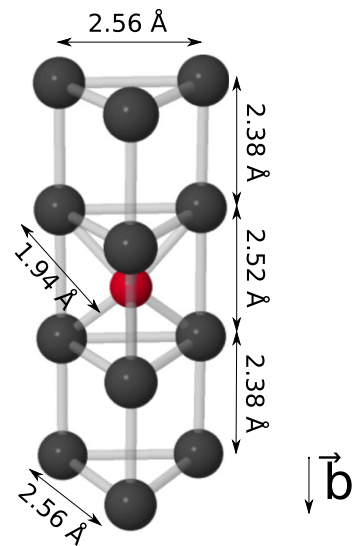

(b)
FIG. 2. (Color online) Relaxed structure of a hard core configuration with regular trigonal prisms of iron atoms (in dark gray) centered on carbon atoms (in red). The core is seen (a) in projection along the Burgers vector and (b) in perspective with the Burgers vector pointing upwards. The carbon atoms are separated by $2 b$ and in (a) iron atoms surrounding the hard core are shown in light gray.

comparable in the hard core and in cementite. Third, in addition to $\mathrm{Fe}_{3} \mathrm{C}$, other metastable carbides, such as $\mathrm{Fe}_{5} \mathrm{C}_{2}$ (Hägg carbide), which form upon tempering of martensitic steels at low temperature [33], also present similar iron trigonal prisms centered on carbon atoms. The same argument applies to $\mathrm{W}(\mathrm{C})$ where tungsten carbide, WC, is also formed of such C-centered prisms of $\mathrm{W}$ atoms [34]. The prism structure resulting from the decoration of the line defect by solute atoms does not alter its dislocation character, and thus its long range elastic field and its interaction with other defects or with an applied stress.

The solute-dislocation interaction energy per solute atom, a function of the solute concentration along the dislocation line, is defined as the energy difference between the situations where the solute atom is in or infinitely separated from the dislocation core. With this definition, negative energies indicate attraction. The reference configurations used in our definition of the interaction energy are the easy core structure for the dislocation and the isolated octahedral interstitial for the solute atom. The carbon-dislocation interaction energy in this prism configuration is $-0.59 \mathrm{eV}$ when carbon atoms are separated by $1 b$, implying a strong attraction between

TABLE I. DFT results for the Fe-Fe and Fe-C distances (in $\AA$ ) for the hard core of the dislocation, the $\mathrm{P}$ position, and the $\mathrm{Fe}_{3} \mathrm{C}$ cementite structure. The carbon atoms are separated by $2 b$ and only the iron atom's first nearest neighbors to the carbon atom are considered. The two $\mathrm{Fe}-\mathrm{Fe}$ distances, $d_{\mathrm{Fe}-\mathrm{Fe}}^{\perp}$ and $d_{\mathrm{Fe}-\mathrm{Fe}}^{\|}$, are perpendicular and parallel to the threefold symmetry axis of the trigonal prism respectively. For comparison, the first and second nearest neighbor distances in the perfect iron lattice are 2.45 and $2.83 \AA$, respectively.

\begin{tabular}{lccc}
\hline \hline & Hard core & $\mathrm{P}$ & $\mathrm{Fe}_{3} \mathrm{C}$ \\
\hline$d_{\mathrm{Fe}-\mathrm{Fe}}^{\perp}$ & 2.44 & 2.56 & $2.45-2.66$ \\
$d_{\mathrm{Fe}-\mathrm{Fe}}^{\|}$ & 2.45 & 2.52 & 2.62 \\
$d_{\mathrm{Fe}-\mathrm{C}}$ & & 1.94 & $1.96-1.99$ \\
\hline \hline
\end{tabular}


carbon atoms and dislocation cores. Upon increasing the distance between solutes, the interaction energy first decreases rapidly to $-0.77 \mathrm{eV}$ for a separation of $2 b$, reflecting a strong repulsion between first neighbor carbon atoms, and then increases slowly, $-0.79 \mathrm{eV}$ for $3 b,-0.75 \mathrm{eV}$ for $4 b$, $-0.74 \mathrm{eV}$ for $5 b$, and $-0.73 \mathrm{eV}$ for $6 b$, due to the energetic cost of transforming the dislocation core from the easy to the hard configuration, either perfect or distorted. The rapid increase, in absolute value, of the interaction energy when the separation distance varies from $1 b$ to $2 b$ and the saturation beyond this distance imply a strong repulsion between carbon atoms, which is, however, mostly limited to the first neighbors. Also, the instability of the $\mathrm{O}^{(2)}$ position means that the capture radius of the screw dislocation extends at least up to its second nearest neighbors.

Additional empirical potential calculations using the embedded atom method (EAM) potential for Fe developed by Mendelev et al. [35] and the EAM potentials for $\mathrm{Fe}-\mathrm{C}$ developed by Hepburn and Ackland [36] and by Becquart et al. [37,38] were performed with the $\mathrm{O}^{(1)}$ and $\mathrm{O}^{(2)}$ configurations. None of these calculations exhibit a spontaneous reconstruction to the prism structure, but predict instead a core spreading. This failure could be related to the inability of the empirical potential to reproduce the low energy of the hard core evidenced in pure iron by DFT [12,14]. Only a distorted prism could be obtained when starting from the DFT prism structure. The Hepburn potential strongly disagrees with the DFT calculations, leading to repulsive carbon-dislocation interaction energy. The Becquart potential agrees qualitatively with the DFT calculations that the carbondislocation interaction is attractive, about -0.1 and $-0.4 \mathrm{eV}$ for carbon-carbon separations of $1 b$ and $2 b$, respectively, and therefore that carbon atoms in first neighbor positions along the dislocation line repel each other.

We now examine the consequences of this strong binding of carbon atoms on the thermal stability of the screw dislocation. Considering only a first nearest neighbor interaction between carbon atoms along the dislocation line, the carbon-dislocation interaction energy per carbon atom calculated by DFT can be expressed as $E_{\text {int }}(1 b)=E_{\text {int }}^{(0)}+$ $\Delta E_{\text {easy-hard }}+V_{\mathrm{CC}}$ and $E_{\text {int }}(2 b)=E_{\text {int }}^{(0)}+2 \Delta E_{\text {easy-hard, where }}$ $E_{\text {int }}^{(0)}$ is the interaction energy of an isolated carbon atom with the hard core, $\Delta E_{\text {easy-hard }}$ the energetic cost to transform a segment of length $b$ from an easy to a hard core, and $V_{\mathrm{CC}}$ the first-neighbor repulsion energy between carbon atoms. From our DFT calculations, we obtain $E_{\text {int }}^{(0)}=-0.84 \mathrm{eV}$, $\Delta E_{\text {easy-hard }}=0.04 \mathrm{eV}$, and $V_{\mathrm{CC}}=0.21 \mathrm{eV}$. Here we have $V_{\mathrm{CC}}=E_{\text {int }}(1 b)-E_{\text {int }}(2 b)+\Delta E_{\text {easy-hard }}$ because $V_{\mathrm{CC}}$ is computed with respect to the hard core, while the interaction energies are relative to the easy core. This strong repulsion is consistent with that obtained by Hatcher et al. in bulk Fe [39]. Then, assuming the carbon atoms at equilibrium, we employ a thermodynamical mean-field model on an effective lattice [40,41], which accounts for configurational entropy, but neglects other sources of entropy, vibrational, electronic, and magnetic. According to the mean-field model, the average carbon-dislocation interaction energy is modeled as

$$
E_{\mathrm{int}}\left(c_{d}\right)=E_{\mathrm{int}}^{(0)}+\frac{\Delta E_{\text {easy-hard }}}{c_{d}}+c_{d} V_{\mathrm{CC}},
$$

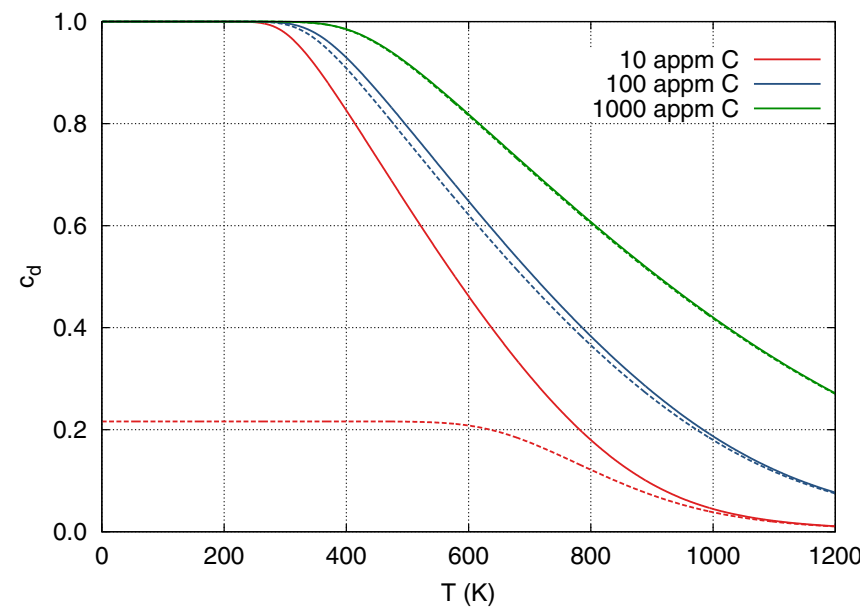

FIG. 3. (Color online) Temperature dependence of the carbon concentration segregated in $\mathrm{P}$ sites for three typical nominal concentrations of carbon and for two dislocation densities, $10^{12} \mathrm{~m}^{-2}$ (solid lines) and $10^{15} \mathrm{~m}^{-2}$ (dashed lines).

where $c_{d}$ is the average carbon concentration on the dislocation core. Minimizing the free energy, we obtain [41]

$$
\frac{c_{d}}{1-c_{d}}=\frac{c_{\text {bulk }}}{1-c_{\text {bulk }}} \exp \left(-\frac{E_{\text {seg }}\left(c_{d}\right)}{k_{B} T}\right),
$$

where the segregation energy is expressed as

$$
E_{\mathrm{seg}}\left(c_{d}\right)=E_{\mathrm{int}}+c_{d} \frac{\partial E_{\mathrm{int}}}{\partial c_{d}}=E_{\mathrm{int}}^{(0)}+2 c_{d} V_{\mathrm{CC}} .
$$

The carbon concentration in the matrix, $c_{\text {bulk }}$, is linked to the nominal concentration of carbon atoms per iron atom, $c_{\text {nom }}$, by matter conservation. In a volume $V$, the number of carbon sites along the dislocation cores is $N_{d}=\rho V / b$, with $\rho$ the dislocation density, while the number of octahedral sites in the matrix is $N_{0}=6 \mathrm{~V} / \mathrm{a}^{3}$, with $a$ the lattice parameter. This leads to the following constraint on the carbon concentrations: $N_{0} c_{\text {bulk }}+N_{d} c_{d}=N_{0} c_{\text {nom }} / 3$, where $N_{d} \ll N_{0}$ and three octahedral sites correspond to one iron atom. With the help of this relation, Eq. (2) can be solved self-consistently to obtain the carbon concentration on the dislocation lines as a function of the nominal carbon concentration and the dislocation density.

Figure 3 shows the temperature dependence of $c_{d}$ for different nominal concentrations ranging from 10 at. ppm, typical of high purity iron, to 1000 at. ppm, representative of low carbon steels, and for a low and a high dislocation density, respectively. The main result is that, unless the carbon concentration is very low and the dislocation density is very high, the dislocation cores are fully saturated by carbon atoms and adopt a hard core configuration up to at least room temperature. Given the diffusion coefficient of carbon atoms in iron [42], the time for carbon transport by diffusion over $1 / \sqrt{\rho}$, the typical distance between dislocations, varies from $10^{5}$ to $10^{8} \mathrm{~s}$ at $300 \mathrm{~K}$ and from 0.02 to $20 \mathrm{~s}$ at $600 \mathrm{~K}$, when the dislocation density varies from $10^{15}$ to $10^{12} \mathrm{~m}^{-2}$. Thus the equilibrium assumption used for carbon atoms in this thermodynamic modeling will be valid in a window of temperature, dislocation density, and strain rate. 
In conclusion, the much-debated stability of the easy core structure appears to be systematic only in pure metals, while the addition of solute atoms may stabilize the hard core configuration because of a low-energy trigonal prism configuration, similar to the building units of $\mathrm{Fe}_{3} \mathrm{C}$ cementite and WC tungsten carbide. Contrary to popular belief, the hard core structure is therefore essential in our understanding of plasticity in bcc metals. The mobility of decorated screw dislocations remains to be investigated in detail, but preliminary DFT calculations for a distance between carbon atoms of $1 b$ show a very strong pinning effect, with the decorated dislocation immobile at $0 \mathrm{~K}$ up to $8 \mathrm{GPa}$, while the classical Peierls stress of the undecorated dislocation is about $1 \mathrm{GPa}$ $[13,14]$. We therefore expect the decorated core to play a central role in yielding and strain aging [3], in agreement with recent in situ experiments, which evidence a locking of the screw dislocations in $\mathrm{Fe}$ around $500 \mathrm{~K}$, and a viscous motion above, resulting from their strong interaction with the carbon atoms [43]. The solute enriched cores may also serve as nuclei for heterogeneous precipitation of a second phase on dislocation cores, such as cementite in $\mathrm{Fe}(\mathrm{C})$ [44], and may lead to a wetting transition at dislocations, similar to wetting of planar interfaces [45].

The authors gratefully acknowledge Dr. F. Ducastelle and Dr. H. Amara (ONERA, France) for pointing out the similarity with the cementite structure. The authors would like to acknowledge support from the GENCI-CINES computer center under Grant No. 2013-096821 and from PRACE (Partnership for Advanced Computing in Europe) for access to the resource MareNostrum III at the Barcelona supercomputing center (DIMAIM project). This work has been carried out within the framework of the EUROfusion Consortium and has received funding from the Euratom research and training programme 2014-2018 under Grant Agreement No. 633053.
[1] Physical Metallurgy, edited by D. E. Laughlin and K. Hono (Elsevier, Amsterdam, 2014), 5th ed.

[2] G. P. M. Leyson, W. A. Curtin, L. G. Hector, Jr., and C. F. Woodward, Nat. Mater. 9, 750 (2010).

[3] A. H. Cottrell and B. A. Bilby, Proc. Phys. Soc., London, Sect. A 62, 49 (1949).

[4] E. Clouet, S. Garruchet, H. Nguyen, M. Perez, and C. S. Becquart, Acta Mater. 56, 3450 (2008).

[5] A. Ishii, J. Li, and S. Ogata, PLoS One 8, e60586 (2013).

[6] L. Proville and S. Patinet, Phys. Rev. B 82, 054115 (2010).

[7] D. L. Olmsted, L. G. Hector, Jr., W. A. Curtin, and R. J. Clifton, Modell. Simul. Mater. Sci. Eng. 13, 371 (2005).

[8] S. Ismail-Beigi and T. A. Arias, Phys. Rev. Lett. 84, 1499 (2000).

[9] C. Woodward and S. I. Rao, Phys. Rev. Lett. 88, 216402 (2002).

[10] C. Domain and G. Monnet, Phys. Rev. Lett. 95, 215506 (2005).

[11] E. Clouet, L. Ventelon, and F. Willaime, Phys. Rev. Lett. 102, 055502 (2009).

[12] L. Ventelon, F. Willaime, E. Clouet, and D. Rodney, Acta Mater. 61, 3973 (2013).

[13] M. Itakura, H. Kaburaki, and M. Yamaguchi, Acta Mater. 60, 3698 (2012).

[14] L. Dezerald, L. Ventelon, E. Clouet, C. Denoual, D. Rodney, and F. Willaime, Phys. Rev. B 89, 024104 (2014).

[15] C. R. Weinberger, G. J. Tucker, and S. M. Foiles, Phys. Rev. B 87, 054114 (2013).

[16] L. Romaner, C. Ambrosch-Draxl, and R. Pippan, Phys. Rev. Lett. 104, 195503 (2010).

[17] G. D. Samolyuk, Y. N. Osetsky, and R. E. Stoller, J. Phys.: Condens. Matter 25, 025403 (2013).

[18] D. R. Trinkle and C. Woodward, Science 310, 1665 (2005).

[19] M. Itakura, H. Kaburaki, M. Yamaguchi, and T. Okita, Acta Mater. 61, 6857 (2013).

[20] Y. Zhao and G. Lu, Modell. Simul. Mater. Sci. Eng. 19, 065004 (2011).

[21] G. Kresse and J. Furthmüller, Phys. Rev. B 54, 11169 (1996).
[22] E. Kuramoto, Y. Aono, and K. Kitajima, Scr. Metall. 13, 1039 (1979).

[23] M. K. Miller, Micros. Res. Tech. 69, 359 (2006).

[24] D. Caillard, Acta Mater. 59, 4974 (2011).

[25] J. M. Soler, E. Artacho, J. D. Gale, A. García, J. Junquera, P. Ordejón, and D. Sánchez-Portal, J. Phys.: Condens. Matter 14, 2745 (2002).

[26] P. Giannozzi, S. Baroni, N. Bonini, M. Calandra, R. Car, C. Cavazzoni, D. Ceresoli, G. L. Chiarotti, M. Cococcioni, I. Dabo, A. D. Corso, S. de Gironcoli, S. Fabris, G. Fratesi, R. Gebauer, U. Gerstmann, C. Gougoussis, A. Kokalj, M. Lazzeri, L. Martin-Samos, N. Marzari, F. Mauri, R. Mazzarello, S. Paolini, A. Pasquarello, L. Paulatto, C. Sbraccia, S. Scandolo, G. Sclauzero, A. P. Seitsonen, A. Smogunov, P. Umari, and R. M. Wentzcovitch, J. Phys.: Condens. Matter 21, 395502 (2009).

[27] P. E. Blöchl, Phys. Rev. B 50, 17953 (1994).

[28] G. Kresse and D. Joubert, Phys. Rev. B 59, 1758 (1999).

[29] D. E. Jiang and E. A. Carter, Phys. Rev. B 67, 214103 (2003).

[30] C. Domain, C. S. Becquart, and J. Foct, Phys. Rev. B 69, 144112 (2004).

[31] V. Vitek, Cryst. Lattice Defects 5, 1 (1974).

[32] R. W. G. Wyckoff, Crystal Structures (Interscience, New York, 1964), Vol. 2, p. 112.

[33] C.-B. Ma, T. Ando, D. L. Williamson, and G. Krauss, Metall. Trans. A 14, 1033 (1983).

[34] W. Hume-Rothery and G. V. Raynor, The Structure of Metals and Alloys (The Institute of Metals, London, 1962), Vol. 1, p. 279.

[35] M. I. Mendelev, S. Han, D. J. Srolovitz, G. Ackland, D. Y. Sun and M. Asta, Philos. Mag. 83, 3977 (2003).

[36] D. J. Hepburn and G. J. Ackland, Phys. Rev. B 78, 165115 (2008).

[37] C. S. Becquart, J. M. Raulot, G. Bencteux, C. Domain, M. Perez, S. Garruchet, and H. Nguyen, Comput. Mater. Sci. 40, 119 (2007).

[38] R. G. A. Veiga, C. S. Becquart, and M. Perez, Comput. Mater Sci. 82, 118 (2014). 
[39] N. Hatcher, G. K. H. Madsen, and R. Drautz, Phys. Rev. B 86, 155115 (2012).

[40] J. P. Hirth and J. Lothe, Theory of Dislocations (Wiley, New York, 1982).

[41] G. Tréglia, B. Legrand, F. Ducastelle, A. Saúl, C. Gallis, I. Meunier, C. Mottet, and A. Senhaji, Comput. Mater. Sci. 15, 196 (1999).
[42] J. R. G. da Silva and R. B. McLellan, Mater. Sci. Eng. 26, 83 (1976).

[43] D. Caillard and J. Bonneville, Scr. Mater. 95, 15 (2015).

[44] F. C. Larché, Dislocations in Solids, Vol. 4 (North-Holland, Amsterdam, 1979).

[45] A. P. Sutton and R. W. Ballufi, Interfaces in Crystalline Materials (Oxford University Press, New York, 1995). 